HVCs was particularly pronounced in patients with high-grade tumors, those with tumors larger than $10 \mathrm{~cm}$ in size ( $P=0.001$ for both), and those with truncal or retroperitoneal sarcomas $(P=0.011)$. Multivariate analysis demonstrated treatment at an HVC to be an independent predictor of good outcome.

On the basis of these results, the authors suggest that patients with STS who have large or high-grade tumors should be referred to HVCs only.

Original article Gutierrez JC et al. (2007) Should soft tissue sarcomas be treated at high-volume centers? An analysis of 4,205 patients. Ann Surg 245: 952-958

\section{Adjuvant mitotane prolongs recurrence-free survival in adrenocortical carcinoma}

Mitotane has been widely used as adjuvant therapy in patients with adrenocortical carcinoma; however, the efficacy of this agent remains unclear. Terzolo and colleagues have recently evaluated the role of mitotane in patients with adrenocortical carcinoma who had undergone resection.

This retrospective analysis included 177 patients who had undergone radical surgery between 1985 and 2005. Adjuvant mitotane was administered at four tertiary referral centers to 47 Italian patients according to center-specific policies, while 55 Italian patients treated at four different referral centers did not receive adjuvant mitotane. To minimize potential biases, a second independent group of 75 German patients, who did not receive any therapy after surgery, was included in the analysis. The two groups of Italian patients had similar baseline characteristics; however, the German patients were significantly older and had a higher proportion of stage I or II tumors than did patients in the mitotane group ( $P=0.03$ and $P=0.02$, respectively). The median recurrence-free survival was 42 months for mitotane-treated patients compared with 10 months in the Italian controls $(P<0.001)$ and 25 months in the German controls $(P=0.005)$. The risk of recurrence was higher in both the Italian and German control groups than in the mitotane group (hazard ratios 2.91 and 1.97, respectively). Mitotane-associated adverse events were mainly of grade 1 or 2 . Adverse grade 3 gastrointestinal and neurologic events were observed only in patients treated with high-dose mitotane ( $3-5 \mathrm{mg} /$ day).
These results suggest that adjuvant mitotane therapy after radical resection might prolong recurrence-free survival in patients with adrenocortical carcinoma.

Original article Terzolo M et al. (2007) Adjuvant mitotane treatment for adrenocortical carcinoma. N Engl J Med 356: 2372-2380

\section{Excessive multivitamin use might increase the risk of advanced and fatal prostate cancer}

Multivitamin supplements are taken by $35 \%$ of adults in the US but, alarmingly, epidemiological evidence suggests that multivitamin use might be associated with more rapid progression of prostate cancer. Other data indicate that multivitamin use may protect against initiation of the disease. In a large prospective study, Lawson et al. investigated the association between multivitamin use and the risk of localized, advanced and fatal prostate cancer in 295,344 men enrolled in the $\mathrm{NIH}$-American Association of Retired Persons Diet and Health Study.

All participants were cancer free at enrollment (1995-1996), when information on use of multivitamin and individual supplements during the preceding 12 months was obtained by questionnaire. Prostate cancer was diagnosed in 10,241 participants ( 8,765 localized and 1,476 advanced) during 5 years follow-up. Mortality analysis (6 years of follow-up) identified 179 cases of fatal prostate cancer. After adjustment for prostate cancer risk factors, multivitamin use was not associated with the risk of localized prostate cancer. By contrast, men who reported excessive use of multivitamin supplements (more than seven times per week) had an increased risk of advanced and fatal prostate cancer compared with never users (relative risk 1.32 [95\% Cl 1.04-1.67] and 1.98 [95\% Cl 1.073.66], respectively); concomitant use of some individual supplements seemed to increase the risk further.

Detection bias is unlikely to have distorted the positive association found between excessive multivitamin use and advanced and fatal prostate cancer. These worrying findings require further assessment.

Original article Lawson KA et al. (2007) Multivitamin use and risk of prostate cancer in the National Institutes of HealthAARP Diet and Health study. J Natl Cancer Inst 99: 754-764 